\title{
Genome-wide identification, phylogeny and expression analysis of GRAS gene family in tomato
}

Wei Huang, Zhiqiang Xian, Xia Kang, Ning Tang and Zhengguo Li ${ }^{*}$

\begin{abstract}
Background: GRAS transcription factors usually act as integrators of multiple growth regulatory and environmental signals, including axillary shoot meristem formation, root radial pattering, phytohormones, light signaling, and abiotic/biotic stress. However, little is known about this gene family in tomato (Solanum lycopersicum), the most important model plant for crop species with fleshy fruits.

Results: In this study, 53 GRAS genes were identified and renamed based on tomato whole-genome sequence and their respective chromosome distribution except 19 members were kept as their already existed name. Multiple sequence alignment showed typical GRAS domain in these proteins. Phylogenetic analysis of GRAS proteins from tomato, Arabidopsis, Populus, P.mume, and Rice revealed that SIGRAS proteins could be divided into at least 13 subfamilies. SIGRAS24 and SIGRAS40 were identified as target genes of miR171 using5'-RACE (Rapid amplification of cDNA ends). qRT-PCR analysis revealed tissue-/organ- and development stage-specific expression patterns of SIGRAS genes. Moreover, their expression patterns in response to different hormone and abiotic stress treatments were also investigated.
\end{abstract}

Conclusions: This study provides the first comprehensive analysis of GRAS gene family in the tomato genome. The data will undoubtedly be useful for better understanding the potential functions of GRAS genes, and their possible roles in mediating hormone cross-talk and abiotic stress in tomato as well as in some other relative species.

\section{Background}

Transcription factors (TFs) are important part of the functional genomics. Since the first transcription factor was found in maize [1], a large number of TFs have been proven to participate in various physiological processes and regulatory networks in higher plants. GRAS proteins are named after GAI, RGA and SCR [2-4], the first three functionally identified members in this family. Typically, proteins of this family exhibit considerable sequence homology to each other in their C-terminus, within which motifs including LHR I, VHIID, LHR II, PFYRE and SAW can be recognized in turn [5-7]. In contrast, $\mathrm{N}$-terminus of GRAS family varies in length and sequence, which seems like the major contributor to the functional specificity of each gene $[6,8]$.

\footnotetext{
* Correspondence: zhengguoli@cqu.edu.cn

Genetic Engineering Research Center, School of Life Sciences, Chongqing University, Chongqing 400044, People's Republic China
}

By far, GRAS gene family has been genome-wide explored in several plant species, including Populus, Arabidopsis, rice, Chinese cabbage, Prunus mume, and pine [9-12]. However, only small number of GRAS proteins were functionally characterized, including some members identified in Zea mays, Petunia hybrida, Medicago truncatula, Lilium longiflorum [13-16]. These genes play crucial roles in diverse fundamental processes of plant growth and development. For instance, the most widely known sub-branch of GRAS proteins, which share the amino acid sequence DELLA in their N-terminal region and thus are referred as DELLA proteins, function as repressors of gibberellin signaling [4]. The SCR and SHR, which belong to two different sub-branches of GRAS family, are both involved in radial organization of the root through forming a SCR/SHR complex [17]. Two independent studies demonstrated that endodermisexpressed SCL3 acted as an integrator downstream of the GA/DELLA and SCR/SHR pathways, mediating the 
GA-promoted cell elongation during root development $[18,19]$. Another sub-branch, which contains 4 highly homologous in Arabidopsis, PAT1, SCL5, SCL13, and SCL21, are involved in light signaling pathways. Interestingly, PAT1, SCL5, SCL21 are positive regulators of phytochrome-A signal transduction while SCL13 is mainly participated in phytochrome-B signal transduction [20-22]. Two GRAS proteins, NSP1 and NSP2 can form a DNA binding complex which is essential for nodulation signaling in legumes [23]. MOC1, mainly expressed in the axillary buds, has a pivotal role in controlling rice tillering [24]. Ls and $L A S$, the homologous gene of MOC1 in tomato and Arabidopsis, also act in the axillary meristem initiation of tomato $[25,26]$. In addition, LiSCL is a transcriptional activator of some meiosis-associated genes, participates in the microsporogenesis of the lily anther [16]. HAM mediates signals from differentiating cells for controlling shoot meristem maintenance in the Petunia [14]. And three Arabidopsis orthologs of Petunia HAM, SCL6/SCL6-IV, SCL22/SCL6-III and SCL27/SCL6-II, also known as targets of post-transcriptional degradation by miRNA170/171, have been demonstrated to play an important role in the proliferation of meristematic cells, polar organization and chlorophyll synthesis [27-29].

Tomato (Solanum lycopersicum) is an important crop because of its great nutritive and commercial value, and also a good model plant for fleshy fruit development. With the release of the whole genome sequence of tomato [30], it is very convenient to comprehensive analysis an entire gene family now. To date, transcription factor families like ERF, WRKY, SBP-box, IAA, ARF, and TCP have already been identified in tomato [31-36]. Here, considering the important role of GRAS proteins in plant growth regulation and the lack of information about this gene family in the crop, we describe on the first characterization of the entire GRAS gene family of transcription factors in tomato. The present work identified 53 putative SlGRAS genes, together with analyzing their gene classification, chromosome distribution, phylogenetic comparison and exon-intron organization. In addition, the expression profile analysis of SlGRAS genes by real time qPCR in different stages of vegetative and reproductive development were performed, and their transcript abundance in response to different hormones and abiotic stress treatments were also investigated. This study provides details of GRAS gene family and facilitates the further functional characterization of GRAS genes in tomato.

\section{Results}

Identification and multiple sequence analysis of SIGRAS genes

Phytozome Search Tools (http://www.phytozome.net/ search.php) was performed using keywords search with
"GRAS", and 54 genes were found when searched against the pfam GRAS hidden-Markov model (PF03154). However, one of them, Solyc09g090830.2.1 was excluded because it represented only part of the GRAS domain and was annotated as an BolA-like protein in the Tomato Genome database (ITAG2.4 Release: genomics annotations). Meanwhile, BLASTP analysis using the amino acid (AA) sequences of characterized AtGRAS proteins as queries obtained 51 previously annotated GRAS members in tomato WGS Chromosomes (SL2.50), which were all included in the 53 GRAS genes identified above. Subsequently, online bioinformatics tools, ExPASy-PROSITE (http://prosite.expasy.org/) and TBLASTN of NCBI showed that all sequences contained a GRAS domain, thus further confirmed the authenticity of the identified SlGRAS genes. Taken together, a total of 53 distinct GRAS transcription factors were indentified in tomato genome (Fig. 1 and Additional file 1). All of the 53 tomato GRAS genes were mapped onto the 12 tomato chromosomes and then renamed based on their distributions and relative linear orders among the respective chromosome (Fig. 2), among which, SIDELLA and SlLs were kept as their already existed name, and so did the SlGRAS1 to SlGRAS17, which were previously described by Mayrose et al. [37]. The tomato GRAS genes display uneven distributions across the chromosomes., Chr1 occupies the largest number of GRAS genes $(\mathrm{n}=8), 4,4,5,5,6$, and 6 GRAS genes were found on Chr10, Chr 12, Chr2, Chr11, Chr 6, and Chr7, respectively, and the other 5 chromosomes each have 3 GRAS genes. Besides, there are 15 SlGRAS genes (SlGRAS20, SlGRAS21, SlGRAS22, SlGRAS23, SlGRAS17, SlGRAS8, SlGRAS25, SlGRAS26, SlGRAS30, SlGRAS31, SlGRAS13, SlGRAS35, SlGRAS44, SlGRAS45, SlGRAS46) clustered into seven tandem duplication event regions on tomato chromosome 1 (2 clusters), 2 ( 2 clusters), 5 (1 cluster), 6 (1 cluster) and 10 (1 cluster) (Fig. 2 and Additional file 2). The size of the deduced GRAS proteins varies greatly, ranging from 125 amino acids (SlGRAS35) to 864 amino acids (SlGRAS33). The molecular weight varies from 14 to $98 \mathrm{kDa}$, and the predicted theoretical pI also varies from 4.93 to 9.57 . These facts indicate that different SIGRAS proteins might function in different microenvironments. Most members possess a variable $\mathrm{N}$-termianl and a single highly conserved C-terminal GRAS domain. However, three members (SlGRAS20, SlGRAS29, and SlGRAS35) present their GRAS domains in the $\mathrm{N}$-terminal part, whereas SlGRAS19, contains two GRAS domains. Interestingly, 41 GRAS genes with only one exon were found, which seems like a widespread phenomenon of this gene family observed in many plant species [9-12]. The exon number of other GRAS genes ranged from two to five. More detailed information about each GRAS gene was shown in Fig. 1, including the GRAS gene group name, gene locus 


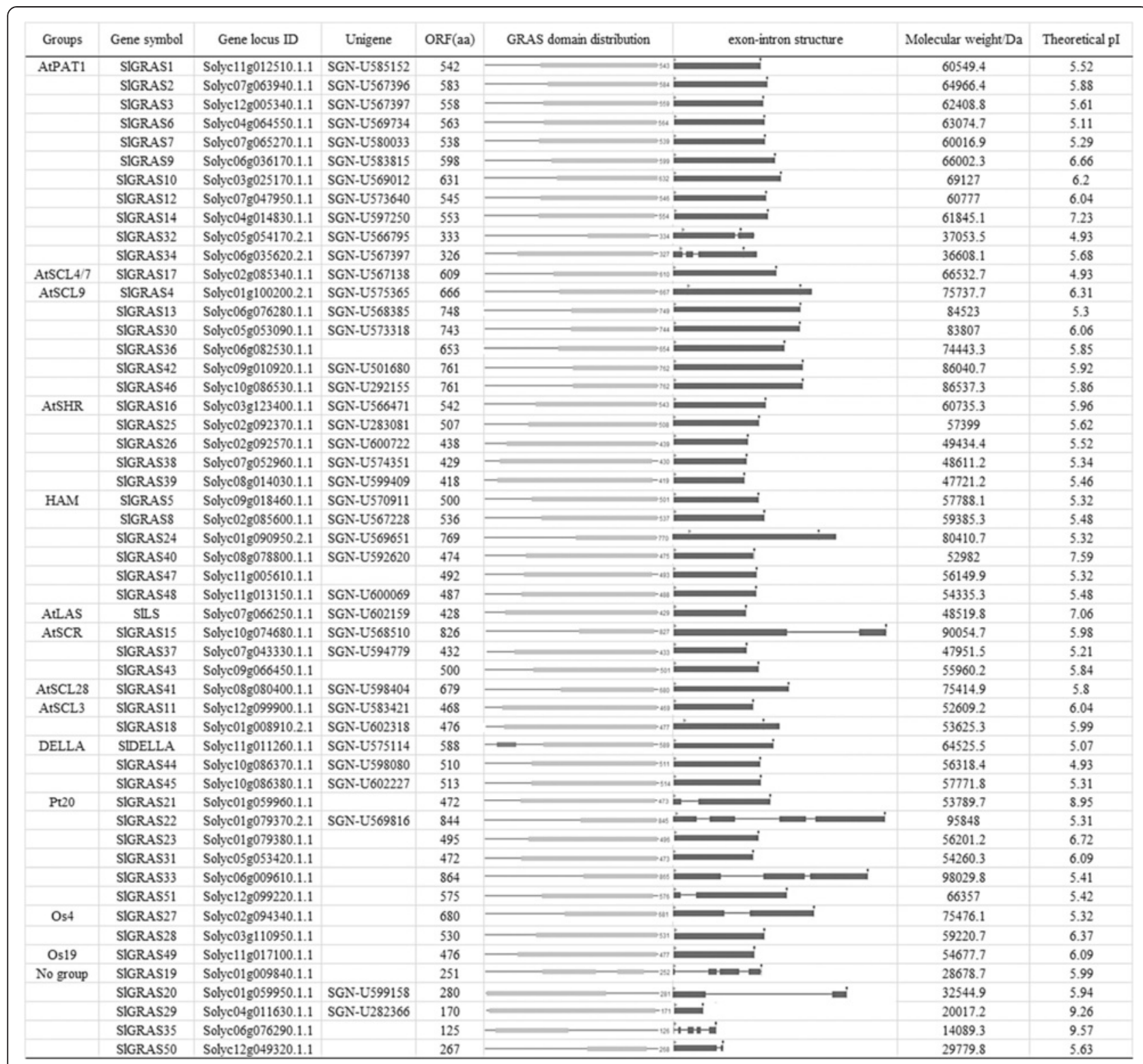

Fig. 1 The information of 53 GRAS transcription factors identified in tomato genome. SIGRAS19, SIGRAS20, SIGRAS29, SIGRAS35, SIGRAS50, whose full amino acid length less than 300 were distributed to "No group" and were excluded from some of the following analyses

number, the length of coding sequences, the schematic plots of GRAS domain, the exon-intron structure, the molecular weight, and the theoretical $\mathrm{pI}$ information.

From the alignment of predicted GRAS domain sequences we found members containing partial GRAS domains with missing motifs, some of which were severely truncated. In tomato, for instance, the GRAS domain of SIGRAS35 could be as short as 85 amino acids, while the typical GRAS domain had a minimum length of about 350 amino acids (e.g., At4g00150, SIGRAS38), thereby 5 non-canonical GRAS proteins (SlGRAS19, SlGRAS20, SlGRAS29, SlGRAS35, SlGRAS50) were excluded from some of the following analyses (multiple sequence alignment and phylogenetic analysis) because of the low reliability by incorporating these fragments. Furthermore, although the multiple sequence analysis showed a low overall identity among the 48 analyzed SIGRAS proteins, the 5 most prominent motifs, including leucine-rich region I (LR I), VHIID, leucine-rich region II (LR II), PFYRE, and SAW could be observed in their GRAS domains (Fig. 3 and Additional file 3).

\section{Phylogenetic analysis and classification of GRAS members from Arabidopsis and tomato}

To uncover the evolutionary history of the GRAS gene family in tomato and to help in their classification, a total 


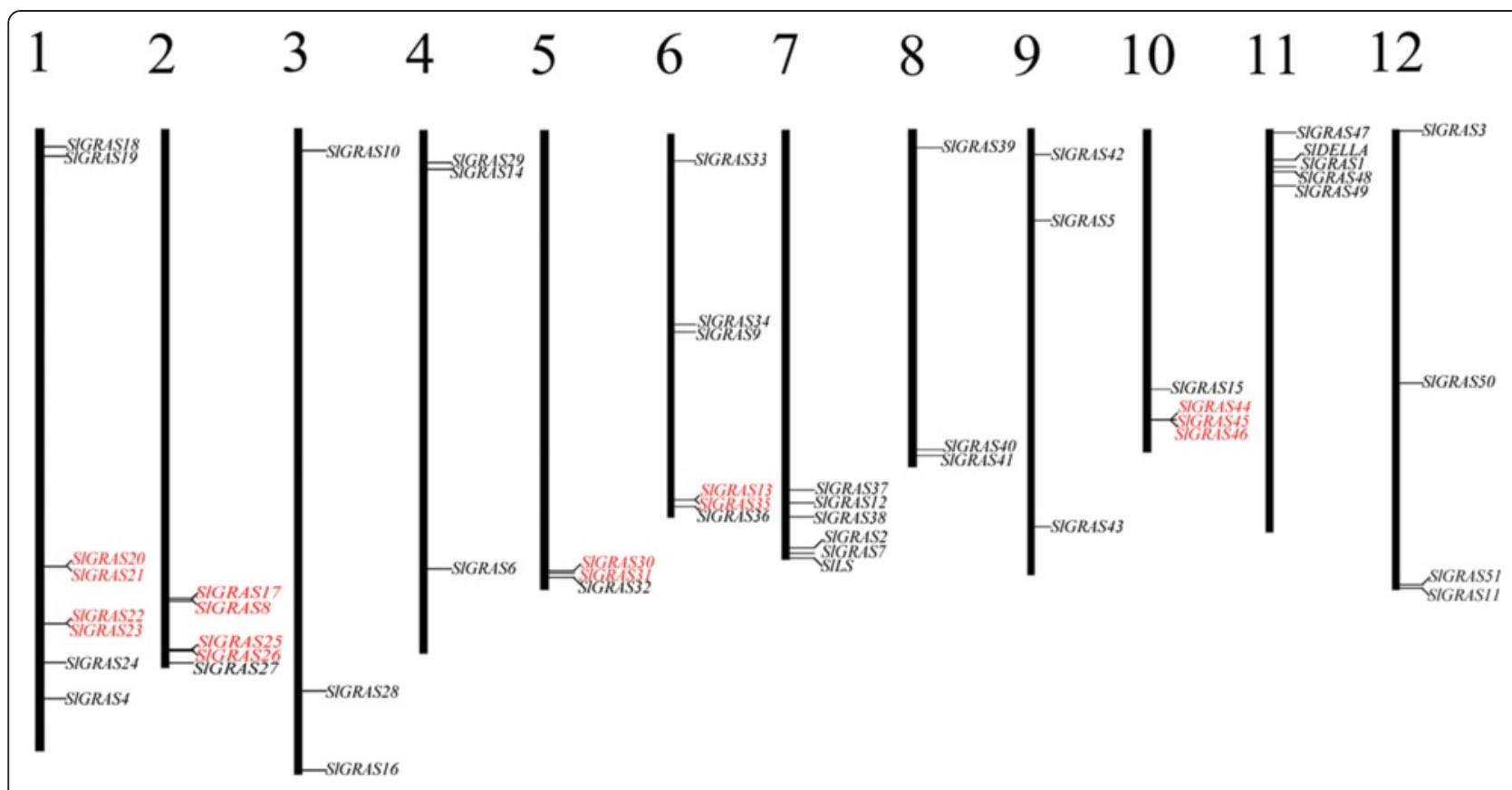

Fig. 2 Positions of GRAS gene family members on the Solanum lycopersicum chromosomes. Tandemly duplicated genes were indicated in red colour

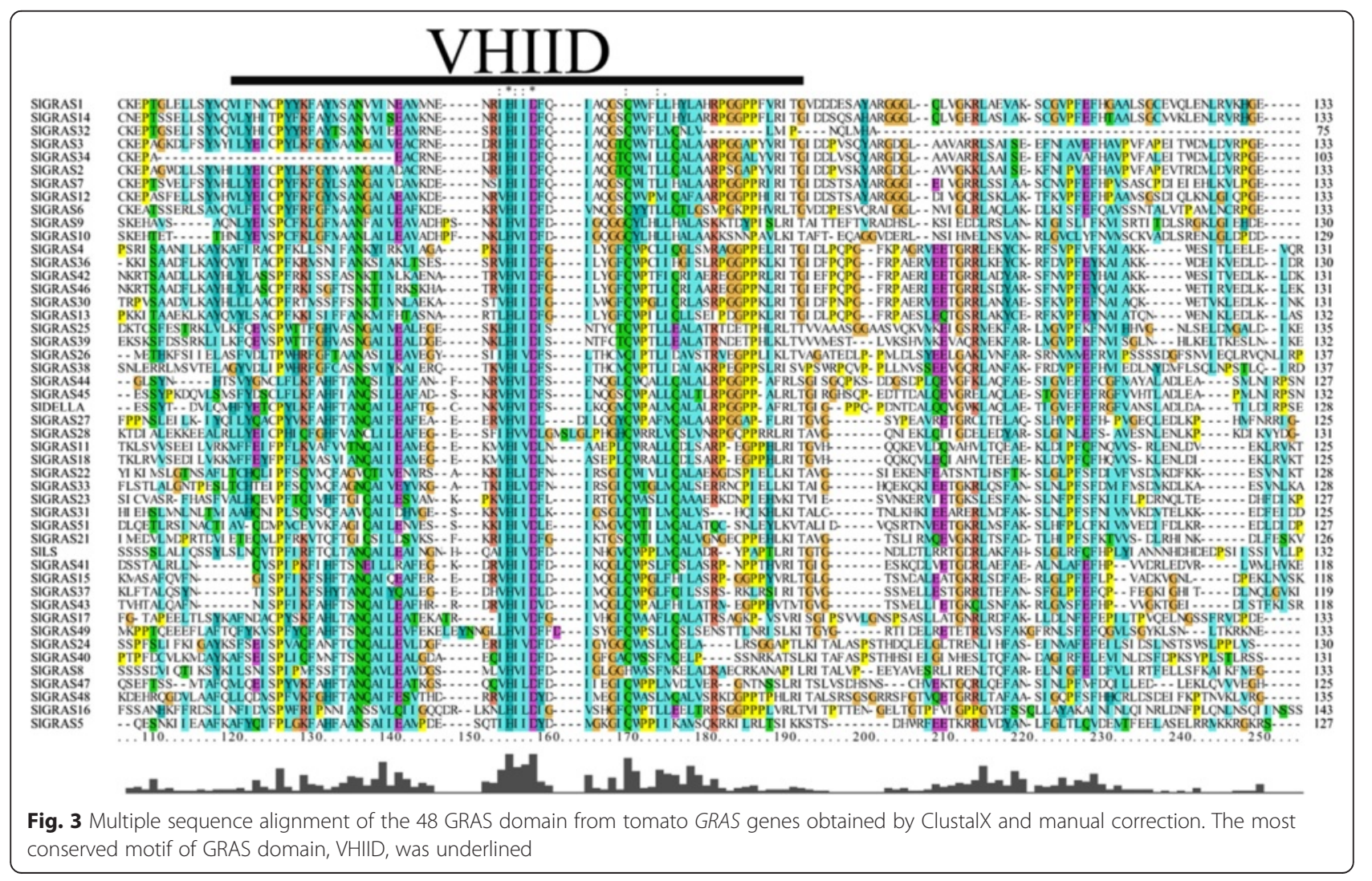


of 124 GRAS proteins, comprising 32 from Arabidopsis, 48 from tomato, 14 from Prunus mume, 14 from Populus, and 16 from Rice, were performed to construct an unrooted phylogenetic tree usingNeighbor-Joining (NJ) method by MEGA6.0 (Fig. 4). Based on the phylogenetic tree, the GRAS proteins could be divided into 13 subfamilies: AtPAT1, AtSCL4/7, AtSCL9, AtSHR, HAM, AtLAS, AtSCR, AtSCL3, AtSCL28,DELLA, Pt20, Os4, and Os19, agree well with the tree made by Liu et al. [9]. It is noteworthy that some GRAS proteins considered to be species-specific in previous publications have homologs in tomato. For example, 6 tomato SlGRAS genes (SlGRAS21, SlGRAS22, SlGRAS23, SlGRAS31, SlGRAS33, SlGRAS33), together with PmGRAS20 and PtGRAS20, belong to
"Pt20" subfamily, which was previously regarded as Populus-specific group [9]. Two (SlGRAS27, SlGRAS28) and one (SlGRAS49) tomato GRAS genes, were clustered into "Os4" and "Os19" subfamily, respectively, which were previously reported as rice-specific protein groups [9]. These three subfamilies did not include any Arabidopsis genes, implying lineage-specific gene loss in Arabidopsis. The other 10 subfamilies harbor GRAS genes from each of the five species with one to eleven SlGRAS genes per group. To date, the functions of the SILS and SIDELLA protein have been clearly illuminated in tomato [25, 38-40]. AtPAT1 subfamily includes 11 members from tomato, two SIGRAS proteins (SIGRAS7 and SIGRAS12) and three SIGRAS proteins (SIGRAS1, SIGRAS14,

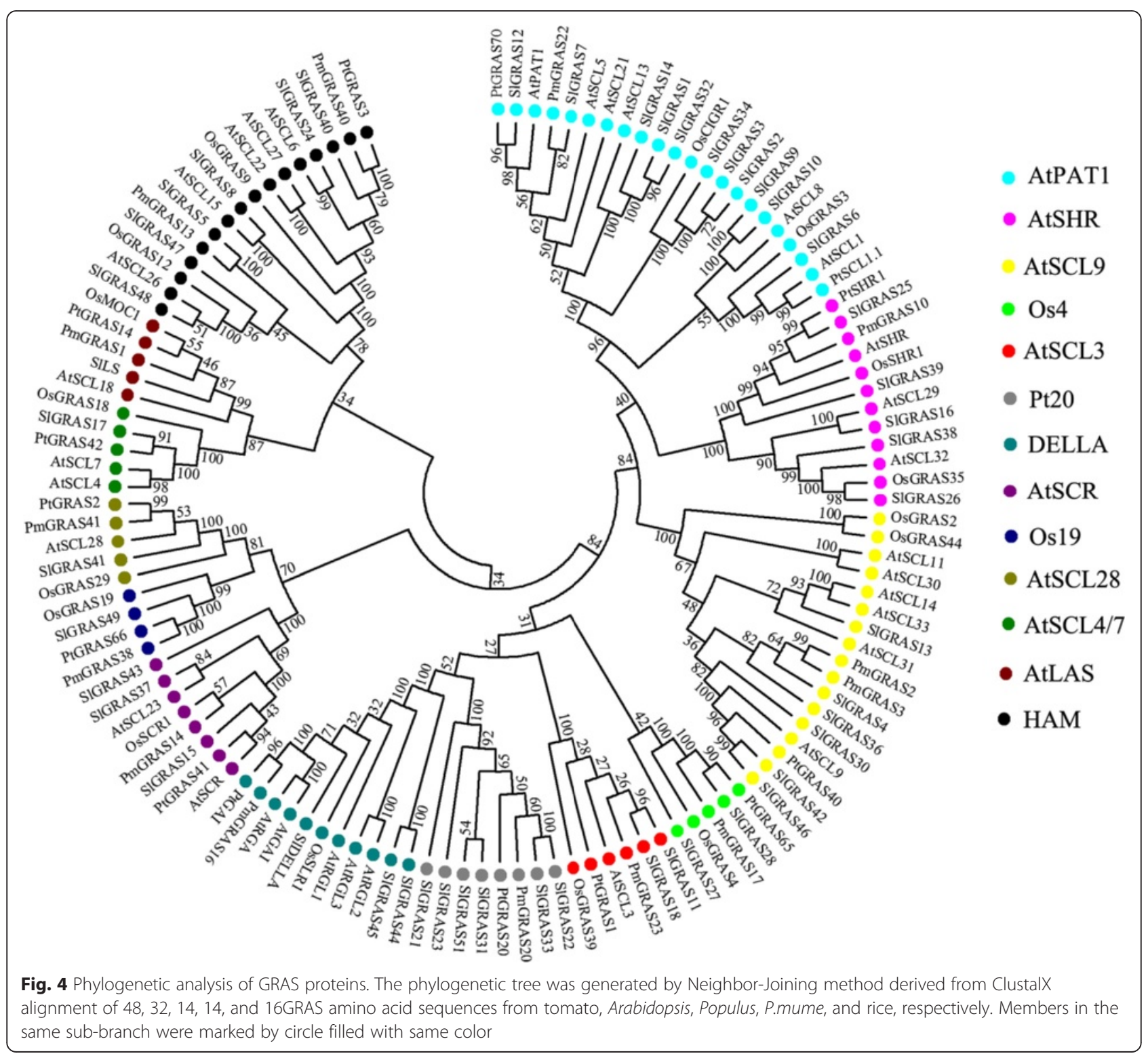


SIGRAS32) have high sequence similarity with AtPAT1 and AtSCL13, respectively, which are associated with phytochrome A and B signaling, respectively [22], suggesting that tomato GRAS homologs might have similar functions in the phytochrome signal transduction. Six proteins (SlGRAS4, SlGRAS13, SIGRAS30, SIGRAS36, and SlGRAS42) are clustered into AtSCL9 subfamily. Although the biological roles of Arabidopsis GRAS proteins in this group are largely unknown, a member of this group in Lilium longiflorum named LiSCL was identified as transcriptional regulator during microsporogenesis [16]. Five (SlGRAS16, SlGRAS25, SlGRAS26, SIGRAS38, and SlGRAS39) and three (SIGRAS15, SlGRAS37, and SlGRAS43) proteins belong to AtSHR and AtSCR subfamily, respectively. Considering the important role of AtSHR and AtSCR proteins in root and shoot radial patterning [17], we predict these homologous genes in tomato may be related to root and shoot development. Two proteins (SlGRAS11 and SlGRAS18) belong to AtSCL3 subfamily, which regulates root cell elongation by integrating multiple signals in Arabidopsis $[18,19]$. SlGRAS41 is the only member of AtSCL28 subfamily in tomato, and a homologous gene identified in rice, OsGRAS29 (also known as DLT), is involved in controlling the plant height of by modulating brassinosteroid signaling [41]. There are 6 SlGRAS proteins (SlGRAS5, SIGRAS8, SIGRAS24, SIGRAS40, SIGRAS47, and SIGRAS 48) clustered into the HAM subfamily. In Arabidopsis, 3 GRAS proteins of this group are posttranscriptionally regulated by miR171 (AtSCL6, 22, 27) [42, 43]. Here, the closest homologs of these Arabidopsis genes in tomato are the two genes, SlGRAS24 and SlGRAS40, both having a putative binding site for SlymiR171. Hence, 5'-RACE was performed to confirm their relationship. As expected, the 5'-RACE products of the predicted size to be generated from cleaved SIGRAS24 and SlGRAS40 template could be amplified. Subsequently, these products were cloned and the sequences of several independent inserts were determined. Sequencing results showed that the complementary sequences of each gene to Sly-miR171 mature sequence as well as the cleavage sites were exactly the same (Fig. 5). Interestingly, in silico analysis (http://plantgrn.noble.org/psRNATarget/) showed that another member of HAM subfamily, SIGRAS8, can also bind Sly-miR171 mature sequence and was predicted to be regulated through translational repression rather than mRNA cleavage, suggesting that a complicated regulatory mechanism of Sly-miR171 and its target genes in tomato.

In addition, to further explore the orthologous relationships of GRAS genes between tomato and other Solanaceae crops, 50 and 30 GRAS genes from potato (Solanum tuberosum) and pepper (Capsicum annuum), respectively, were selected to construct another phylogenetic tree (Additional file 4). We found that almost every member of
SlGRAS genes (except for SlGRAS17) has its homologous gene(s) in either or both of potato and pepper genome, suggesting that the evolutional conservation and closer homology relationship among GRAS genes in closely related species.

\section{Expression analysis of SIGRAS genes in different tissues and organs}

To investigate the potential functions of SlGRAS genes during tomato development, their expression patterns were carried out in different tissues including root, stem, leaf, bud, anthesis flower and three stages of fruit development using qRT-PCR. In the qPCR analysis, genes exhibiting $\mathrm{Ct}$ value $>36$ were treated as non-expressors. As shown in Figs. 6 and 10a, a total of 45 SlGRAS gene transcripts were obtained, while 8 other SlGRAS genes could not be detected because of their low expression levels or might be pseudogenes. It is apparent that the expression levels in different tissues vary widely among the tomato GRAS genes, as well as among different tissues for individual GRAS genes. Of them, 23, 10, and 8 genes were found exhibit the highest expression in stems, anthesis flowers, and roots, respectively. During fruit development, generally higher transcript abundance can be observed in immature fruits than mature fruits, which suggests that those genes might relate to early fruit development. Nevertheless, several genes show dramatic increase at the breaker stage compared to the immature stage. For example, SlGRAS38, SlGRAS35, and SlGRAS47 display relatively strong and specific expression during fruit ripening, indicating that they might have functional significance during the onset of ripening.

A large number of SlGRAS genes demonstrate relatively high expression in flowers, suggesting the important role of these genes in such tissues. Given that many GRAS proteins are involved in regulating the gibberellic acid (GA) response, one of the key plant hormones during fruit set $[44,45]$, we analyzed the expression profiles of SlGRAS genes during the flower-to-fruit transition process (Figs. 7 and 10b). Of all the $40 S I G R A S$ genes identified, 16 genes exhibite higher expression in stamen while the transcripts of 12 genes are more abundant in ovary tissues, indicating functional specialization among GRAS gene family members in tomato floral organs, at least in stamen and ovary. The data show that most of SlGRAS genes undergo a drastic change in their mRNA levels either or both in stamens and ovaries, suggesting that the GRAS family members play different roles during pollination/fertilization.

\section{Expression analysis of SIGRAS genes in response to hormone treatments}

Plant hormones have been extensively studied for their roles in the regulation of various aspects of plant 


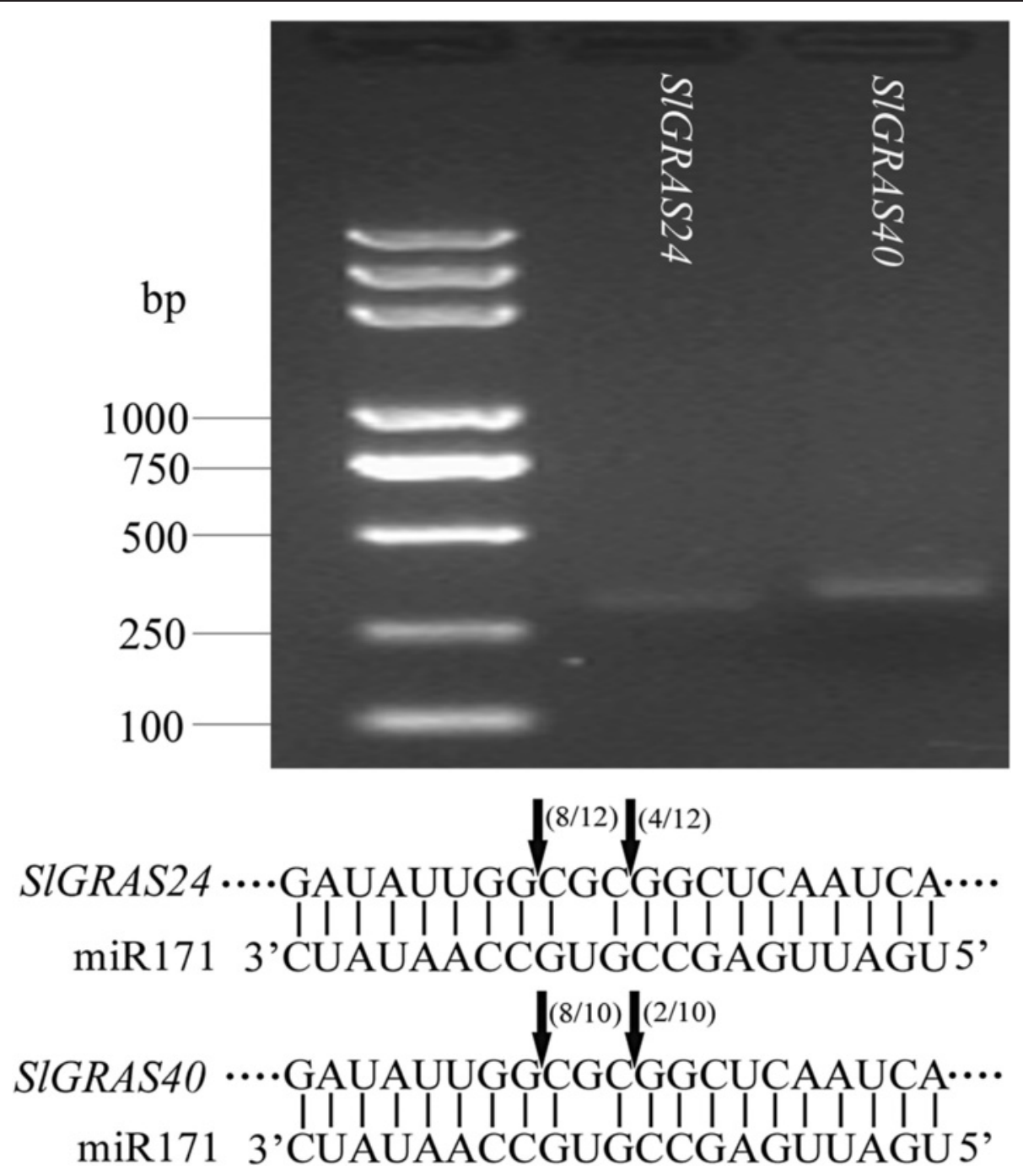

Fig. 5 Cleavage sites of miR171 at complementary sequences of SIGRAS24 and SIGRAS40 determined by 5'-RACE. The electrophoretogram shows the PCR products representing the 3'-cleavage fragments that were cloned and sequenced for each gene. Both SIGRAS24 and SIGRAS40 were cleaved between $10^{\text {th }}$ and $11^{\text {th }}, 13^{\text {th }}$ and $14^{\text {th }}$ nt of mature miR171 sequence (arrows)

development. In this study, hormone treatments resulted in a wide variety of SlGRAS gene expression profiles (Figs. 8 and 10c). The expression levels of 39 GRAS genes detected vary significantly in response to different hormone treatments as well as in different tissues in response to an individual hormone treatment, suggesting that the SlGRAS genes have differences in signal-selectivity not only among different hormones but also among different tissues of tomato seedlings. In ethephon (Eth) treatment, 15 and 12 SlGRAS genes were obviously induced and inhibited, respectively. Of them, the most up-regulated gene was SlGRAS26 in roots, and the most down-regulated gene was SlGRAS36 in shoots. Similarly, GA treatment led to 10 and 9 SlGRAS genes were obviously induced and inhibited, respectively, the most up-regulated gene was SlGRAS26 in roots, while the most down-regulated gene was SlGRAS36 in roots. In IAA treatment, 6 and 17 SlGRAS were significantly induced and inhibited, respectively, and SIGRAS4 and SlGRAS14 in roots were found to be most up- and down-regulated, respectively. As for SA treatment, 20 and 9 SIGRAS genes showed dramatic increase and decrease, respectively, SlGRAS34 and SlGRAS37 in roots went through the largest increase and decrease, respectively. Notably, several genes even demonstrated opposite expression in roots and shoots when responding to the same hormone treatment. For instance, SlGRAS3 was up-regulated in shoots in response to Eth, GA3 and IAA treatments, while down-regulated in roots. Similar expression patterns were found in SlGRAS18, SlGRAS26, SlGRAS41, SlGRAS45, and SlGRAS46. The results suggest the complicated regulatory mechanism of these genes in response to hormone treatments in tomato. Taken together, these expression 


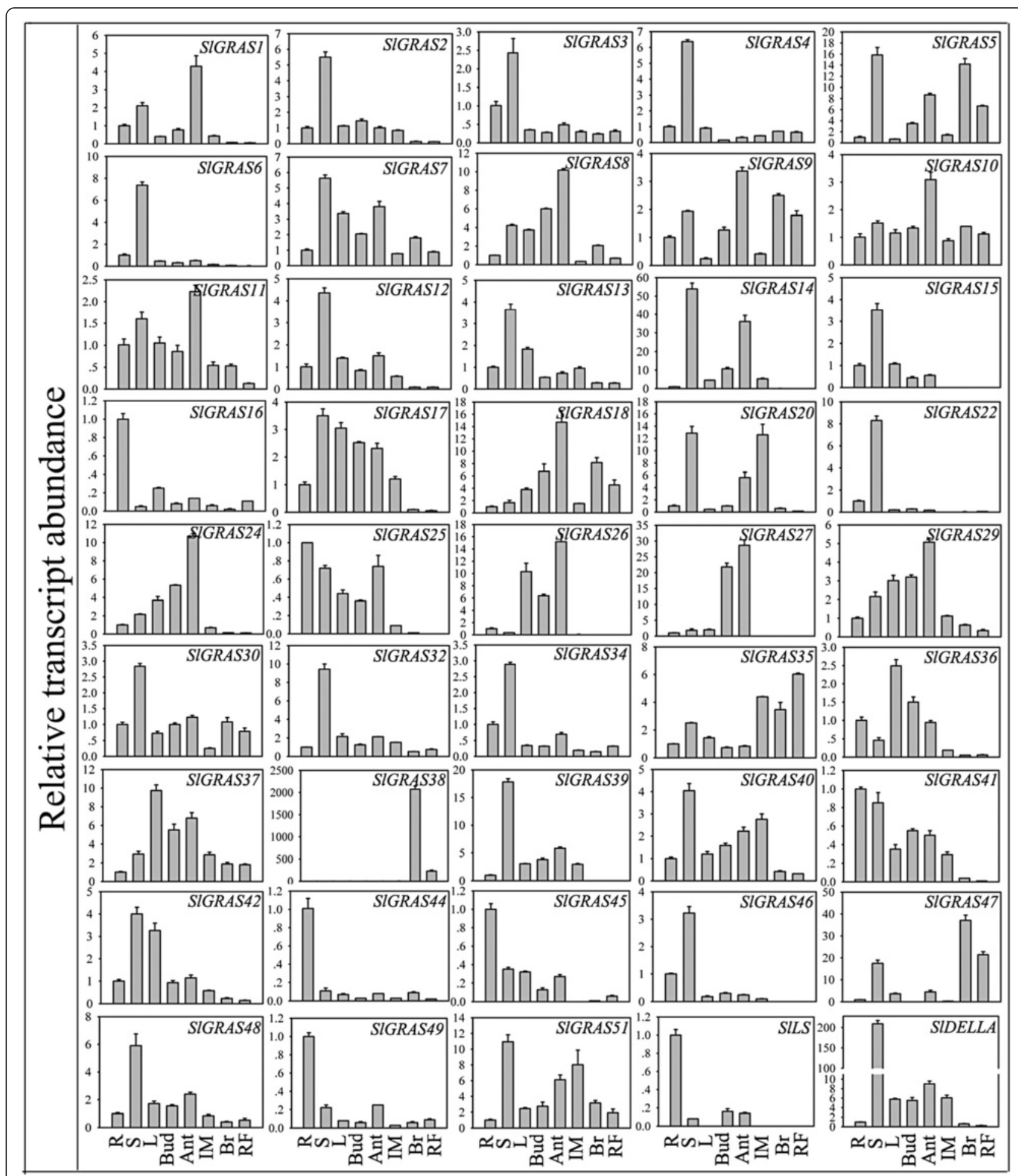

Fig. 6 The expression profiles of 45 SIGRAS genes analyzed using APCR during eight stages of development. Y-axis represents relative expression values and X-axis represents stages of development as follows: R root, S stem, L leaf, Bud bud flower, Ant anthesis flower, IM immature green stages, Br breaker stage, and RF red ripe stage of fruit development. The expression data of root were normalized to 1. Error bars show the standard error between three replicates performed 


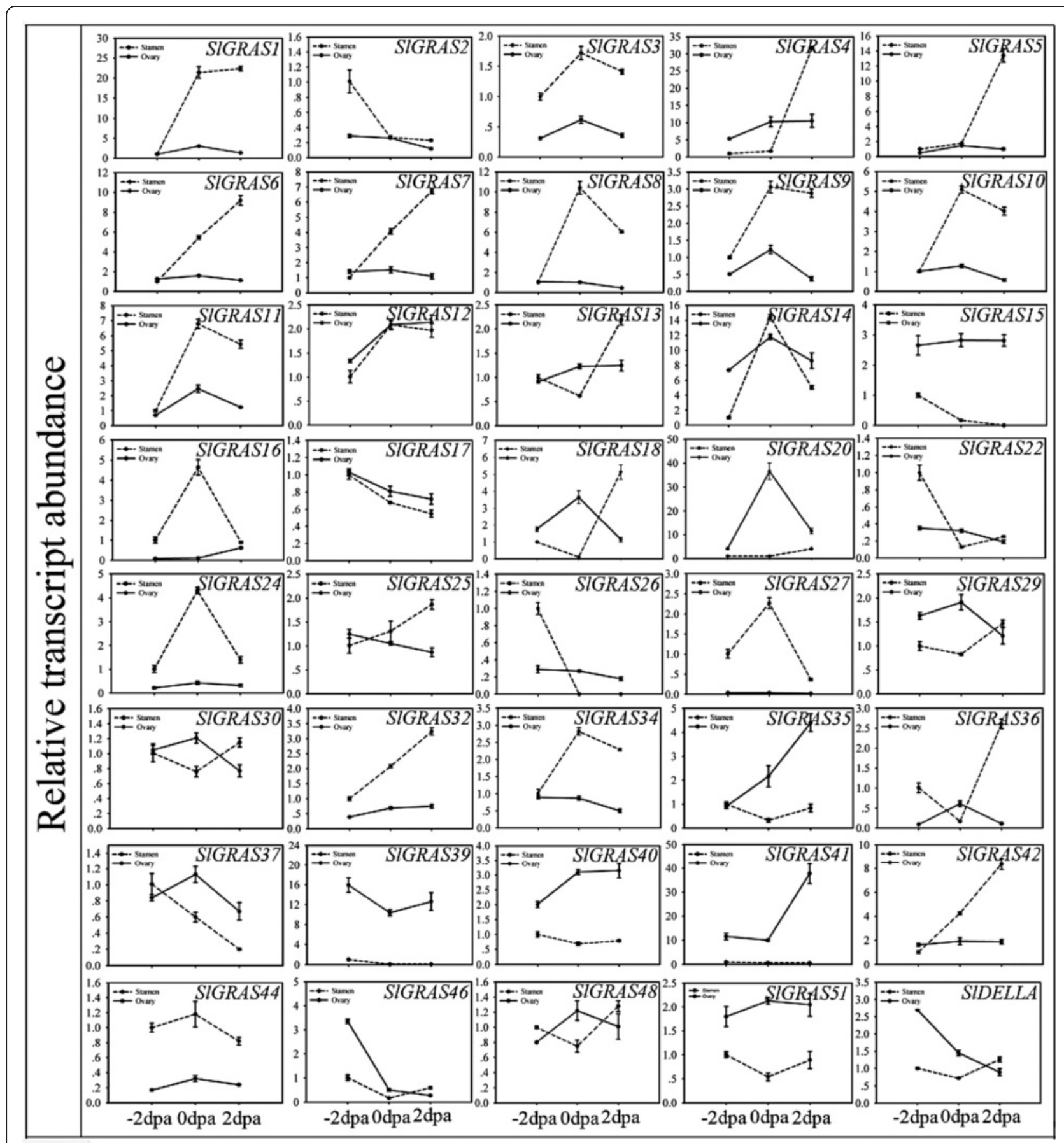

Fig. 7 Expression patterns exhibited by 40 SIGRAS family genes during fruit-set stage of tomato. The X-axis represents 3 different stages, -2 dpa 2 days before anthesis, 0 dpa the first day of anthesis, 2 dpa 2 days post anthesis. Solid lines depict the expression patterns of ovaries while dotted lines stand for stamens. The expression data of $-2 \mathrm{dpa}$ stamens were normalized to 1. Error bars show the standard error between three replicates performed

variations indicate that the SlGRAS gene family members were collectively regulated by a broad range of hormonal signals. Thus it is reasonable to speculate that those relevant genes might play pivotal roles in the cross-talk of hormones and should be candidates for further research in the field.
Expression analysis of SIGRAS genes in response to abiotic treatments

To further assess the functions of SlGRAS genes that may be involved in plant defenses to abiotic stresses, we analyzed the expressions of SlGRAS genes in response to salt, drought, cold, heat, osmotic and oxidative stress 


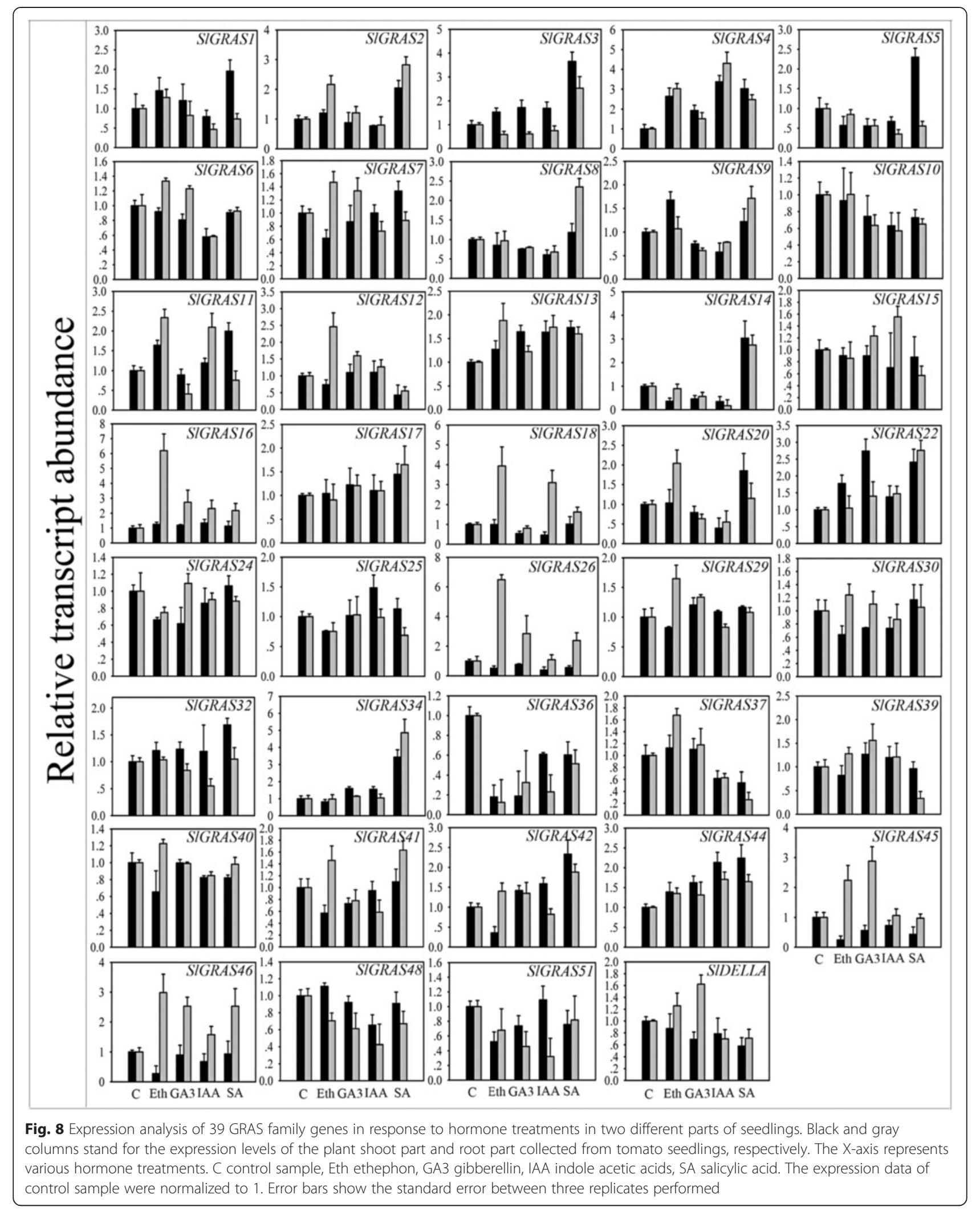


(Fig. 9 and 10d). Although only two genes (SlGRAS26, SlGRAS36) were found to be hyperresponsive to all treatments, all the analyzed genes exhibited differential expression in response to at least one abiotic stress treatments.
Overall, in the six stressed conditions, a total of 30 SIGRAS genes were significantly induced, implying their putative roles in stress tolerance. 18 and 13 SlGRAS genes showed obviously increase and decrease under salt treatment,

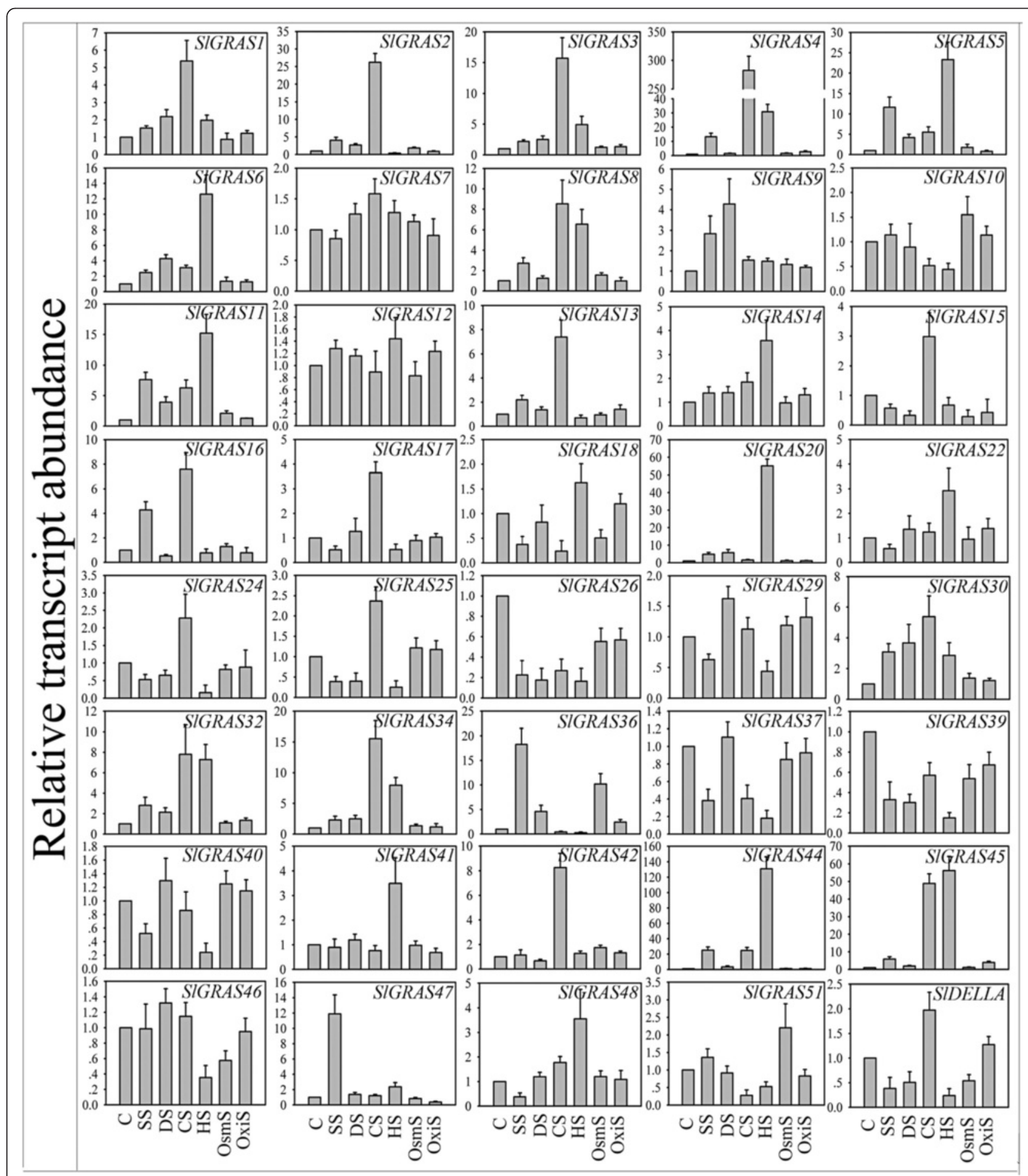

Fig. 9 Expression analysis of 40 GRAS family genes in response to abiotic treatments. The X-axis represents different abiotic stresses. C control sample, SS salt stress, DS drought stress, CS cold stress, HS heat stress, OsmS osmotic stress, OxiS oxidative stress. The expression data of control sample were normalized to 1. Error bars show the standard error between three replicates performed 


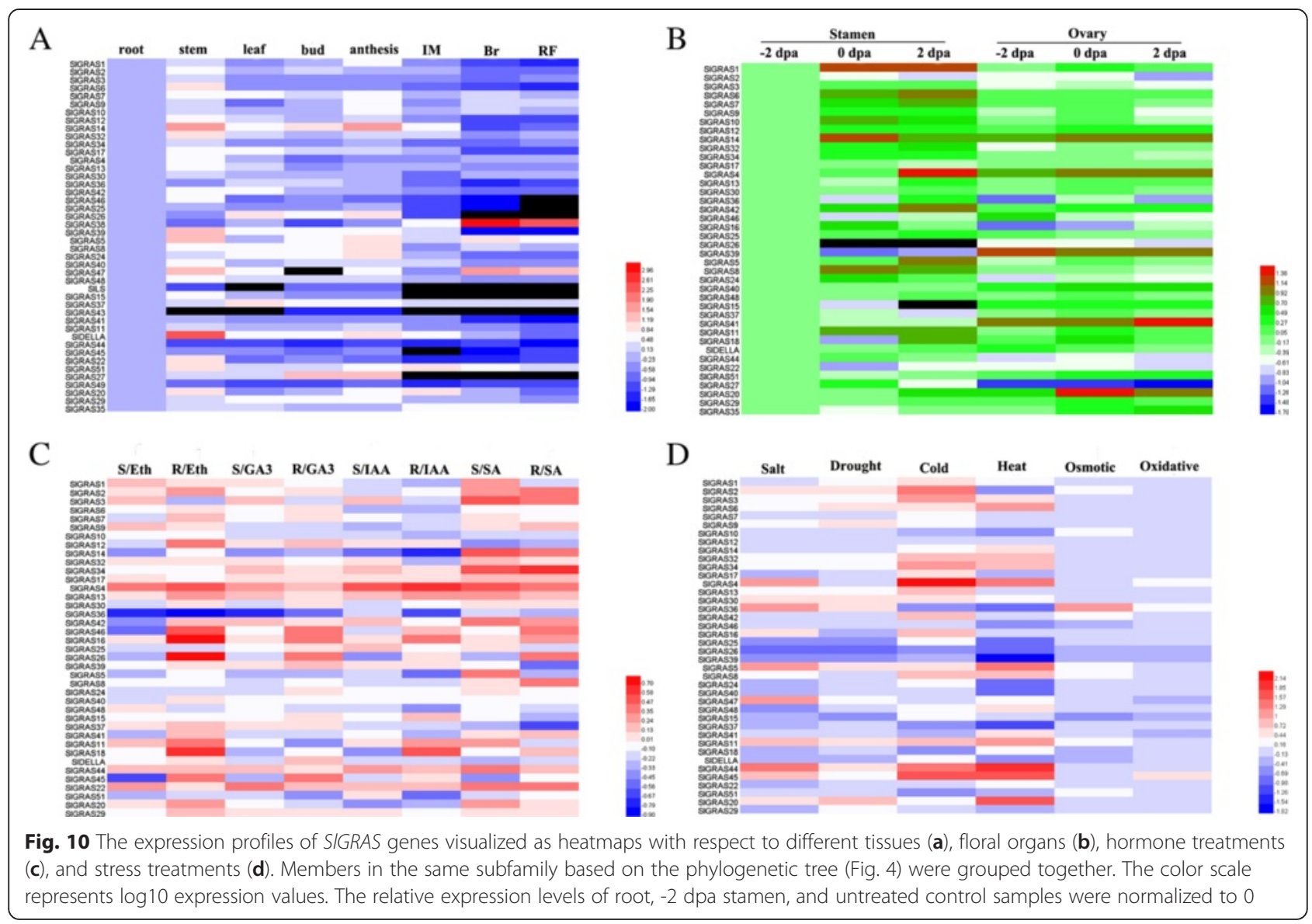

among which, SlGRAS44 and SlGRAS26 underwent the greatest mRNA levels change, respectively. Similarly, 17 genes were up-regulated and 7 genes were down-regulated following drought treatment with greatest change in SlGRAS20 and SlGRAS26, respectively. Intriguingly, most members showed strong sensitivity toward heat (30 SlGRAS genes) and cold treatments (27 SlGRAS genes), and the largest differential expression usually observed when responding to one of these two treatments. For example, the transcript accumulation of SlGRAS4 exhibited more than 250 -folds change during cold stress compared to that in the control plants and SlGRAS20 showed more than 50- folds change in response to heat treatment. By contrast, not too much change observed in most SlGRAS genes when osmotic and oxidative stresses were carried out. These data show the potential of some SlGRAS genes for enhancing adversity resistant capacity, especially considering that the tomato is an extremely temperature-sensitive crop.

\section{Discussion}

To date, several attempts have been made to group members of GRAS family into subfamilies that reflect their evolutionary relationships [6-12]. These dendrograms were in substantial agreement though some fine-tunings. The bioinformatic analysis of GRAS proteins showing higher similarity within the same species indicates that gene duplications have occurred after the split among these lineages. Compared to Arabidopsis, larger number of GRAS proteins arisen in tomato suggests more gene duplications events or higher frequency of the retaining copies after duplication in tomato. Taken the tandem duplication events as example, 2/34, 10/45, 15/53, 17/60, 40/106 GRAS genes were identified as tandem duplicated genes in Arabidopsis [6], P.mume [11], tomato (Fig. 2), rice [6], and Populus [9], respectively, further validating that the duplication events are the most common mechanism contributing to the rapid expansion of GRAS gene family members in different species. Meanwhile, the exon-intron organization analysis showed that $77.4 \%$ of SlGRAS genes were intronless in tomato (Fig. 1), with proportions $82.2 \%, 67.6 \%, 55 \%$ and $54.7 \%$ in P.mume, Arabidopsis, rice and Populus, respectively [9-12]. The high percentage of intronless genes in GRAS gene family in plant implies the close evolutionary relationship of GRAS proteins. Apart from GRAS gene family, intronless genes are also enriched in some other large gene families, such as F-box transcription factor gene family [46], DEAD box RNA helicases [47], and small auxin-up RNAs (SAUR) gene family [48]. Generally, intronless genes 
are archetypical in the prokaryotic genomes, and there are three explanations for the formation of the intronless genes in eukaryotic genomes: horizontal gene transfer from ancient prokaryotes, duplication of existing intronless genes, and retroposition of intron-containing genes [49]. Zhang et al. [50] recently reported the origin of plant GRAS genes from prokaryotic genomes of bacteria by horizontal gene transfer. That might be the reason of the abundant intronless genes in the GRAS gene family, which is likely to be its prokaryotic origin followed by extensive duplication events in the evolutionary history.

Intrinsically disordered proteins (IDPs) are highly abundant in eukaryotic proteomes and important for cellular functions. An IDR (intrinsically disordered region) within an IDP often undergoes disorder-to-order transitions upon binding to various partners, allowing an IDP to recognize and bind different partners at various binding interfaces $[8,51,52]$. By computational and bioinformatics tools, Sun et al. [8] demonstrated that the GRAS proteins are intrinsically disordered. One of the distinguishing features of GRAS proteins is its variable N-terminal, which is predicted to contain MoRFs (molecular recognition features), short interaction-prone segments that are located within IDRs and are able to recognize their interacting partners by undergoing disorder-to-order transitions upon binding to these specific partners [51, 52]. In tomato, except a few uncanonical GRAS proteins, multiple sequence analysis of tomato GRAS proteins showed that most members in this family possess a highly variable $\mathrm{N}$-terminal domain, indicating the functional versatility of this gene family in tomato. Highly conserved C-terminal domains (GRAS domain) were observed in most SIGRAS proteins. Generally, Leucine-rich regions I (LR I) and II (LR II) flank the VHIID motif to form a LR I-VHIID-LR II pattern present in most GRAS proteins. It has been widely and experimentally confirmed for many GRAS proteins that the LRI-VHIID-LRII pattern or individual motifs within the pattern are used for interactions with protein partners $[17,23,51-54]$.

Due to the functional diversity of GRAS genes, many members of this gene family need to be further functionally characterized. The expression patterns of SlGRAS genes here could help us to assess their possible functions. 8 SlGRAS genes were undetectable in any tissues/organs suggests a tendency to degenerate those genes after gene duplication or the lost of their functions during evolution. On the whole, the expression patterns vary greatly among different members even between those orthologous pair genes (SlGRAS1 and SlGRAS32, SlGRAS11 and SlGRAS18, SlGRAS42 and SlGRAS46) (Fig. 6). Previously, expression profiles of GRAS genes in Populus and P.mume also demonstrated rather broad expression patterns across a variety of tissues, not only among subfamilies but members in the same clade $[9,11]$. These results suggest that GRAS genes may undergo neo-functionalization or subfunctionalization in many higher plant species. Yet still, some GRAS genes with extremely high sequence identity (SlGRAS1 and SlGRAS14, SlGRAS2 and SlGRAS3, SlGRAS7 and SlGRAS12, SlGRAS9 and SlGRAS10) (Fig. 6) exhibited conserved expression patterns, implying their retention by genetic redundancy and selection for their contributions to the robustness of the genetic network. SlGRAS25,SlGRAS39 and SlGRAS15 with high mRNA levels in roots and stems suggests conserved functions with their homologous gene AtSHR [17] and AtSCR [55], which are involved in root and shoot radial patterning in Arabidopsis. The strong ovary-preferential expression of SlGRAS41 during flower-fruit transition suggests its potential role in fruit development by modulating brassinosteroid signaling [45]. The homologs of AtSCL3 (SlGRAS11, SlGRAS18) displayed high mRNA levels in anthesis flowers, indicating that they may exert new functions during pollination/fertilization by modulating GA signaling $[18,19]$. Our results have proved that SIGRAS24 and SlGRAS40 can be cleaved by miR171 (Fig. 5), one of the most conserved miRNAs in plants, suggesting that they may have similar functions with their homologous genes characterized in other species such as Arabidopsis [43]. However, the expression patterns of SlGRAS24 and SIGRAS4O in tomato are largely different, which suggests that the complicated and widespread functions of the miR171-GRASs regulatory networks in tomato. Noticeably, according to the Supplementary Table 75 of Tomato Genome Consortium [27], there are 14 SlGRAS genes (SlGRAS1, SlGRAS2, SlGRAS8, SlGRAS9, SlGRAS12, SlGRAS13, SlGRAS14, SlGRAS17, SlGRAS18, SlGRAS24, SlGRAS32, SlGRAS38, SlGRAS40, SlGRAS48) were differentially expressed from mature green stage fruits to breaker stage fruits. Our results are consistent with the above data, suggesting the pivotal roles of these genes during fruit ripening. Two of them, SIGRAS18 and SlGRAS38, predominantly expressed in breaker and red ripening stage fruits, have been reported as target genes of RIN [56, 57], which is key transcriptional regulator during fruit ripening. Moreover, the spatio-temporal expression patterns revealed that the majority members of SlGRAS identified presented sharply increase or decrease upon pollination/fertilization either or both in stamen and ovary (i.e., SlGRAS8, SlGRAS11, SlGRAS14, SlGRAS16, SlGRAS18, SlGRAS20, SlGRAS24, SlGRAS27, SlGRAS36) (Fig. 7), indicating their potential active roles during ovary and anther development. Considering the relationship between GRAS genes and GA signaling, we speculate that members of this gene family involve in mediating GA responses during flower-to-fruit transition.

Plant growth and development are regulated by a chemically and structurally diverse group of hormones. Many 
known growth and development responses to hormones are due to modulation of gene expression, and these responses are among the best characterized to date [58]. In general, hormones control the expression of genes by regulating the abundance of two types of gene regulatory proteins, transcription factors and transcriptional repressors. To our knowledge, the relationship between GRAS proteins and hormones remain scarce, apart from the widely known gibberellin [3, 54], only a few reports mentioned some members involved in auxin and brassinosteroid signal transduction [41, 59, 60]. Among four hormones conducted here, auxin (Indole 3 -acetic acid, IAA) is involved in almost all aspects of plant growth and development, from embryogenesis to senescence, from root tip to shoot tip [61]. Gibberellic acid also regulates a diverse array of developmental processes such as seed development and germination, organ elongation and control of flowering time [62]. Ethylene and salicylic acid play important roles in biotic stresses [63], while ethylene is also the key regulator during fleshy fruit ripening [45]. It has been reported that BnSCL1, a GRAS protein identified in Brassica napus, showed differential dose response to auxin in shoots and roots [59]. The current results demonstrated that the majority of SIGRAS genes detected here displayed distinct changes following different hormone treatments, and some of them even exhibited opposite trends in roots and shoots, suggesting that GRAS transcription factors regulate gene expression by modulating phytohormone signaling through complicated networks (Fig. 8). Additionally, several studies have revealed that GRAS genes play potential regulatory roles in stress responses. PeSCL7, a member of GRAS genes from poplar, was regarded useful for engineering drought- and salttolerant trees [64]. Over-expression of a BnLAS gene in Arabidopsis thaliana could increase its drought tolerance [65]. The DELLA protein was proved to be involved in many abiotic stresses such as low temperature, phosphate starvation, and high NO concentration [66-68]. As for the evidence of GRAS proteins in the regulation of plant defence responses in tomato: transcripts corresponding to GRAS genes in resistant tomato plants infected with virulent phytopathogenic bacteria were different $[69,70]$. Furthermore, the expression analysis by qRT-PCR showed that several tomato GRAS genes were associated with plant disease resistance and mechanical stress response [36]. Many transcription factor families have been shown to display stress-responsive gene expression with significant overlap in response to various stress treatments, indicating the cross correlation upon signaling pathways involved in various stresses. The induction of SIGRAS genes in response to more than one stress treatments in the present work highlights the wide involvement of GRAS genes in environmental adaptation (Fig. 9). We observed that SlGRAS genes showed larger accumulation under salt, cold, and heat treatments compared to other three treatments, suggesting that the SlGRAS gene family members might play more important roles in response to these three stress conditions. Combined analysis of all qPCR data (Figs. 6, 8, 9 and 10), we found that four highly homologous genes belonging to AtPAT subfamily (SlGRAS2, SlGRAS3, SlGRAS7, SlGRAS34) exhibit similar expression levels when responding to hormone and abiotic treatments, implying that these genes may be also involved in hormone signaling and stress response. Consistently, two genes of this subfamily from rice, CIGR1 and CIGR2, were reported to be gibberellin and stress related [71]. SlGRAS36 was significantly decreased in response to all hormone treatments while obviously increased in its mRNA levels upon four abiotic stresses. Likewise, SlGRAS4 was induced by all hormone treatments, and the strong upregulation of its transcripts under cold stress suggests the great potential for cold stress tolerance. Interestingly, both SIGRAS36 and SIGRAS4 share strong sequence similarity to AtSCL14, a GRAS transcription factor that is essential for the activation of stress-inducible promoters [41]. A homologous gene of AtSCL14 from rice, OsGRAS23, is involved in drought stress response through regulating expression of stressresponsive genes [72]. Thus, we deduce that SIGRAS36 and SIGRAS4 may play important role in eliciting stress responsive genes in tomato. Besides, several SIGRAS genes were dramatically regulated under both hormone and abiotic stress treatments, indicating the coordinate response of these two determinants.

\section{Conclusions}

Although some classical functions of GRAS transcription factors have already been characterized in several plant species, more members of the GRAS family in agricultural crops, especially in those with fleshy fruits, remain to be further studied. In this work, 53 GRAS transcription factors were indentified in tomato. The information generated about the structure of SIGRAS proteins will shed light on their functional analysis. The comparative, phylogenetic, and expression analyses of GRAS members will be useful to comprehensive functional characterization of the GRAS gene family, and to better understanding their possible roles in mediating hormone cross-talk and abiotic stress. After all, the data shown here should be taken into consideration in future studies for genetic improvements of agronomic traits and/or stress tolerance in tomato and probably other Solanaceae plants.

\section{Methods}

\section{Plant materials and growth conditions}

Tomato plants (Solanum lycopersicum cv. Micro-Tom) were grown on soil in greenhouse with suitable conditions: $14 / 10 \mathrm{~h}$ light/dark cycle, $25 / 20{ }^{\circ} \mathrm{C}$ day/night temperature and $60 \%$ relative humidity, and the plant 
nutrient solution were irrigated once per week. Roots, stems, and leaves were collected on two-month-old plants, flowers (bud, anthesis) and fruit (immature, breaker stage, and red fruit) were harvested at the proper time. Stamens and ovaries were collected 2 days before anthesis $(-2 \mathrm{dpa})$, the first day of anthesis $(0 \mathrm{dpa})$, and 2 days post anthesis ( $2 \mathrm{dpa})$, respectively. All tissues were collected from six well-grown plants between 9:00 a.m. and 10:00 a.m. and thoroughly mixed, then frozen in liquid nitrogen immediately, and each tissues/ organs were sampled for three independent times.

\section{Identification of tomato GRAS genes}

At first, we used "GRAS" as a key word and the S.lycopersicum genome was chosen as initial queries, a total of 54 putative GRAS genes were obtained from the Phytozome database (http://www.phytozome.net). Meanwhile, systematic BLAST homology searches using amino sequence of the 32 AtGRAS proteins obtained from the National Center for Biotechnology Information (NCBI) were performed on all sequences in the International Tomato Annotation Group Release 2.4 tomato proteins (2.40) (BLASTP, $E$ value $\leq 1 \times 10^{-5}$ ) and tomato WGS chromosomes $(2.50)\left(\right.$ TBLASTN, $E$ value $\left.\leq 1 \times 10^{-5}\right)$ (SGN http:// solgenomics.net/tools/blast/). Taken together, 53 potential GRAS genes were identified from the currently available genomic databases. Subsequently, online bioinformatics tools, ExPASy-PROSITE (http://prosite.expasy.org/) and TBLASTN of NCBI (http://blast.st-va.ncbi.nlm.nih.gov/ Blast.cgi?PROGRAM=tblastn\&PAGE_TYPE=BlastSearch\& LINK_LOC=blasthome) were used to further confirm the presence of GRAS domain in resulting sequences.

\section{Bioinformatic analyses of tomato GRAS genes}

The 53 putative GRAS genes were renamed according to their chromosomal location except 19 members were kept as their already existed name (SlGRAS1-SlGRAS17, SIDELLA, SlLS). The functional domain distribution and exon-intron structures of the GRAS proteins were obtained from Phytozome (http://www.phytozome.net). The tandemly duplicated genes were defined as an array of two or more SlGRAS genes with Smith-Waterman alignment e values $\leq 1 \times 10^{-25}$ in the range of $350-\mathrm{kb}$ distance, as proposed by Lehti-Shiu et al. [73]. We downloaded the GRAS protein sequences of Populus, rice, and P.mume according to two previous publications $[11,12]$, and at least one gene of each subfamily was selected based on the phylogenetic trees. Then, together with all 32 Arabidopsis AtGRAS proteins and 48 tomato SIGRAS proteins, the multiple sequence alignment were performed using the ClustalX2.0 program using the default settings. A phylogenetic tree based on the alignment was constructed using MEGA6.0 by the NJ (neighbour-joining) method with the bootstrap test replicated 1000 times.

\section{Modified 5'-RACE to identify the slicing sites of SIGRAS24 and SIGRAS4O}

The 5'-Full RACE kit (TaKaRa, JAPAN) was used for RNA ligase-mediated rapid amplification of cDNA ends (RLM-RACE) assay according to the manufacture's specification. Briefly, total RNAs were extracted from the seedlings of wild-type tomato, and Poly(A) mRNA was directly ligated to the 5'-RACE adaptor (60 nucleotides). The oligo(dT) primer was used to prime cDNA synthesis with reverse transcriptase. PCR was performed according to Tm of each GSP primers (Additional file 5), which were designed at the predicted 3' products of complementary site of mature miRNA sequence. Finally, the PCR products were purified, cloned into pEASY-Blunt Cloning vector (Transgene) and were sent to sequencing for each product.

\section{Hormone and abotic stress treatments}

For hormone treatment, 12-day-old tomato seedlings were soaked in liquid MS medium with $20 \mu \mathrm{M}$ ethephon (Eth), $20 \mu \mathrm{M}$ gibberellin (GA3), $20 \mu \mathrm{M}$ indole acetic acid (IAA), $20 \mu \mathrm{M}$ salicylic acid (SA) for $3 \mathrm{~h}$, respectively. Roots and shoots of treated samples were harvested separately. Seedlings soaked in liquid MS medium without any hormone were used as control.

About one-month-old tomato plants were subjected to various abiotic stress treatments. For cold or heat treatment, tomato plants which were grown in green house were transferred to a cold chamber maintained at $4 \pm 1{ }^{\circ} \mathrm{C}$ or in an incubator at $42 \pm 1{ }^{\circ} \mathrm{C}$, respectively. Salt, osmotic and oxidative stress treatments were carried out by spraying leaves with $200 \mathrm{mM} \mathrm{NaCl}, 100 \mathrm{mM}$ mannitol and $100 \mathrm{mM}$ hydrogen peroxide. Leaves were sampled at $6 \mathrm{~h}$ post treatment and untreated plants were used as controls. The drought treatment consisted of withholding water for up to 15 days. Well-watered plants were maintained as controls by watering plants daily.

At each treatment, materials from six separate seedlings/ plants were combined to form one sample, and all of the treatment experiments were performed in three independent times. All these samples were frozen in liquid nitrogen immediately and stored at $-80^{\circ} \mathrm{C}$ until RNA extraction.

\section{RNA isolation and real-time quantitative PCR analysis}

Total RNA was extracted using TRIzol reagent (Invitrogen, USA) according to the manufacturers' instruction. RNA integrity was verified by $1.2 \%$ agar gel electrophoresis and the RNA concentration was measured using NanoDrop 1000 (Thermo, USA). The PrimeScript ${ }^{\mathrm{m}}$ RT reagent Kit with gDNA Eraser (TaKaRA, JAPAN) was used to remove any genomic DNA contamination and the first strand 
cDNA synthesis following the manufacturers' protocol. Approximately $2 \mu \mathrm{g}$ of RNA was used for each $20 \mu \mathrm{L}$ reaction. Real-time quantitative PCR was conducted using SsoAdvanced Universal SYBR Green Supermix (BIORAD, USA) on a CFX96 Touch ${ }^{\text {me }}$ Real-Time PCR Detection System (BIO-RAD, USA). Each reaction mixture contained $10 \mu \mathrm{l}$ SYBR Green Supermix, $1 \mu \mathrm{l}$ cDNA template, $0.5 \mu \mathrm{l}$ each primer, and $8 \mu \mathrm{l}$ sterile distilled $\mathrm{H}_{2} \mathrm{O}$. The PCR amplification cycle was as follows: $95{ }^{\circ} \mathrm{C}$ for $30 \mathrm{~s}, 40$ cycles at $95{ }^{\circ} \mathrm{C}$ for $5 \mathrm{~s}$, and $58{ }^{\circ} \mathrm{C}$ for $20 \mathrm{~s}$. Melting curve analysis was performed ranging 60 to $95{ }^{\circ} \mathrm{C}$ to verify the specificity of the amplicon for each primer pairs. Relative fold differences were calculated based on the comparative $\mathrm{Ct}$ method using the $2^{-\Delta \Delta \mathrm{Ct}}$ method with the SIUBI as an internal reference gene. All the primers for $\mathrm{qPCR}$ were designed based on the reference sequence obtained from the tomato WGS chromosomes 2.50 (Additional file 5).

\section{Availability of supporting data}

Phylogenetic data (alignments and phylogenetic trees) supporting the results of this article have been deposited in TreeBASE respository and is available under the URL http://purl.org/phylo/treebase/phylows/study/

TB2:S18045.

\section{Additional files}

Additional file 1: The nucleotide and protein sequence information of all 53 SIGRAS genes. The amino acid sequence of GRAS domain of each protein was indicated in red. (PDF $372 \mathrm{~kb}$ )

Additional file 2: Tandem duplication events in the 53 SIGRAS genes. (PDF $111 \mathrm{~kb}$ )

Additional file 3: Multiple sequence alignment showed the other 4 most prominent motifs of GRAS domain: LR I, LR II, PFYRE, SAW, respectively. (PDF $202 \mathrm{~kb}$ )

Additional file 4: Phylogenetic analysis of GRAS proteins of tomato, potato, and pepper. The phylogenetic tree was generated by Neighbor-Joining method derived from ClustalX alignment of 48, 50, and 30 GRAS amino acid sequences from tomato, potato, and pepper, respectively. (PDF $291 \mathrm{~kb}$ )

Additional file 5: Primers used in this work. (PDF $159 \mathrm{~kb}$ )

\section{Competing interests}

The authors declare that they have no competing interests.

\section{Authors' contributions}

WH, ZGL and ZQX designed the study. WH collected the datasets from databases and analyzed the data, then prepared the original draft the manuscript. WH, ZQX and XK extracted the total RNA and did the qRT-PCR experiments. WH and NT preformed the 5'-RACE experiment. All authors read and approved the final manuscript.

\section{Acknowledgements}

This work was supported by grants from the National High Technology Research and Development Program of China (2012AA101702), National Basic Research Program of China (2013CB127101), the National Natural Science Foundation of China $(31071798,31272166)$, the Committee of Science and Technology of Chongqing (2011BA1024), the Fundamental Research Funds for the Central Universities (CDJXS10231118).
Received: 9 March 2015 Accepted: 11 August 2015

Published online: 25 August 2015

\section{References}

1. Paz-Ares J, Ghosal D, Wienand U, Peterson PA, Saedler $H$. The regulatory c1 locus of Zea mays encodes a protein with homology to myb proto-oncogene products and with structural similarities to transcriptional activators. EMBO J. 1987;12:3553-8.

2. Di Laurenzio L, Wysocka-Diller J, Malamy JE, Pysh L, Helariutta Y, Freshour G, et al. The SCARECROW gene regulates an asymmetric cell division that is essential for generating the radial organization of the Arabidopsis root. Cell. 1996;86:423-33.

3. Peng J, Carol P, Richards DE, King KE, Cowling RJ, Murphy GP, et al. The Arabidopsis GAl gene defines asignaling pathway that negatively regulates gibberellin responses. Genes Dev. 1997;11:3194-205.

4. Silverstone AL, Ciampaglio CN, Sun T. The Arabidopsis RGA gene encodes a transcriptional regulator repressing the gibberellins signal transduction pathway. Plant Cell. 1998;10:155-69.

5. Pysh LD, Wysocka-Diller JW, Camilleri C, Bouchez D, Benfey PN. The GRAS gene family in Arabidopsis: sequence characterization and basic expression analysis of the SCARECROW-LIKE genes. Plant J. 1999;18:111-9.

6. Tian CG, Wan P, Sun SH, Li JY, Chen MS. Genome-wide analysis of the GRAS gene family in rice and Arabidopsis. Plant Mol Biol. 2004;54:519-32.

7. Bolle C. The role of GRAS proteins in plant signal transduction and development. Planta. 2004;218:683-92.

8. Sun $\mathrm{XL}$, Xue $\mathrm{B}$, Jones WT, Rikkerink E, Dunker AK, Uversky VN. A functionally required unfoldome from the plant kingdom: intrinsically disordered $\mathrm{N}$-terminal domains of GRAS proteins are involved in molecular recognition during plant development. Plant Mol Biol. 2011;77:205-23.

9. Liu X, Widemer A. Genome-wide comparative analysis of the GRAS gene familyin Populus, Arabidopsis and Rice. Plant Mol Biol Rep. 2014;32:1129-45.

10. Song X, Liu T, Duan W, Ma Q, Ren J, Wang Z, et al. Genome-wide analysis of the GRAS gene family in Chinese cabbage (Brassica rapa ssp. pekinensis). Genomics. 2014;103:135-46.

11. Lu JX, Wang T, Xu ZD, Sun LD, Zhang QX. Genome-wide analysis of GRAS gene family in Prunus mume. Mol Genet Genomics. 2015;290:303-17.

12. Abarca D, Pizarro A, Hernandez I, Sanchez C, Solana SP, Amo AD, et al. The GRAS gene family in pine: transcript expression patterns associated with the maturation-related decline of competence to form adventitious roots. BMC Plant Bio. 2014;14:354.

13. Lim J, Helariutta Y, Specht CD, Jung J, Sims L, Bruce WB, et al. Molecular analysis of the SCARECROW gene in maize reveals a common basis for radial patterning in diverse meristems. Plant Cell. 2000;12:1307-18.

14. Stuurman J, Jäggi F, Kuhlemeier C. Shoot meristem maintenanceis controlled by a GRAS gene mediated signal from differentiating cells. Genes Dev. 2002;16:2213-8.

15. Kim GB, Nam YW. A novel GRAS proteins gene MtSymSCL1 plays a role in regulating nodule number in Medicago truncatula. Plant Growth Regul. 2013;71:77-92.

16. Morohashi K, Minami M, Takase H, Hotta Y, Hiratsuka K. Isolation and characterization of a novel GRAS gene that regulates meiosis-associated gene expression. J Biol Chem. 2003;278:20865-73.

17. Cui HC, Levesque MP, Vernoux T, Jung JW, Paquette AJ, Gallagher KL, et al. An evolutionarily conserved mechanism delimiting SHR movement defines a single layer of endodermis in plants. Science. 2007:316:421-5.

18. Zhang ZL, Ogawa M, Fleet CM, Zentella R, Hu JH, Heo JO, et al. SCARECROW-LIKE 3 promotes gibberellin signaling by antagonizing master growth repressor DELLA in Arabidopsis. Proc Natl Acad Sci USA. 2011;108:2160-5

19. Heo JO, Chang KS, Kim IA, Lee MH, Lee SA, Song SK, et al. Funneling of gibberellin signaling by the GRAS transcription regulator SCARECROW-LIKE 3 in the Arabidopsis root. Proc Natl Acad Sci USA. 2011;108:2166-71.

20. Bolle C, Koncz C, Chua NH. PAT1, a new member of the GRAS family, is involved in phytochrome A signal transduction. Genes Dev. 2000;14:1269-78

21. Torres-Galea P, Huang LF, Chua NH, Bolle C. The GRAS protein SCL13 is a positive regulator of phytochrome-dependent red light signaling, but can also modulate phytochrome A responses. Mol Genet Genomics. 2006;276:13-30. 
22. Torres-Galea P, Hirtreiter B, Bolle C. Two GRAS proteins, SCARECROW-LIKE2 and PHYTOCHROME A SIGNAL TRANSDUCTION1, function cooperatively in phytochrome A signal transduction. Plant Physiol. 2013;161:291-304.

23. Hirsch S, Kim J, Munoz A, Heckmann AB, Downie JA, Oldroyd GED. GRAS proteins form a DNA binding complex to induce gene expression during nodulation signaling in Medicago truncatula. Plant Cell. 2009;21:545-57.

24. Li XY, Qian Q, Fu ZM, Wang YH, Xiong GS, Zeng DL, et al. Control of tillering in rice. Nature. 2003:422:618-21.

25. Schumacher K, Schmitt T, Rossberg M, Schmitz G, Theres K. The Lateral suppressor (LS) gene of tomato encodes a new member of the VHIID protein family. Proc Natl Acad Sci USA. 1999;96:290-5.

26. Greb T, Clarenz O, Schafer E, Muller D, Herrero R, Schmitz G, et al. Molecular analysis of the LATERAL SUPPRESSOR gene in Arabidopsis reveals a conserved control mechanism for axillary meristem formation. Genes Dev. 2003;17:1175-87.

27. Schulze S, Schafer BN, Parizotto EA, Voinnet O, Theres K. LOST MERISTEMS genes regulate cell differentiation of central zone descendants in Arabidopsis shoot meristems. Plant J. 2010;64:668-78.

28. Engstrom EM, Andersen CM, Gumulak-Smith J, Hu J, Orlova E, Sozzani R, et al. Arabidopsis Homologs of the Petunia HAIRY MERISTEM Gene Are Required for Maintenance of Shoot and Root Indeterminacy. Plant Physiol. 2011;155:735-50

29. Ma ZX, Hu XP, Cai WJ, Huang WH, Zhou X, Luo Q, et al. Arabidopsis miR171-Targeted Scarecrow-Like Proteins Bind to GT cis-Elements and Mediate Gibberellin-Regulated Chlorophyll Biosynthesis under Light Conditions. Plos Genetics. 2014;10:e1004519.

30. Tomato Genome Consortium. The tomato genome sequence provides insights into fleshy fruit evolution. Nature. 2012;485:635-41.

31. Sharma MJ, Kumar R, Solanke AU, Sharma R, Tyagi AK, Sharma AK. Identification, phylogeny, and transcript profiling of ERF family genes during development and abiotic stress treatments in tomato. Mol Genet Genomics. 2010;284:455-75.

32. Huang SX, Gao YF, Liu JK, Peng XL, Niu XL, Fei ZJ, et al. Genome-wide analysis of WRKY transcription factors in Solanum lycopersicum. Mol Genet Genomics. 2012;287:495-513.

33. Salinas M, Xing SP, Hohmann S, Berndtgen R, Huijser P. Genomic organization, phylogenetic comparison and differential expression of the SBP-box family of transcription factors in tomato. Planta. 2012;235:1171-84.

34. Audran-Delalande C, Bassa C, Mila I, Regad F, Zouine M, Bouzayen M. Genome-wide identification, functional analysis and expression profiling of the AUXIAA gene family in tomato. Plant Cell Physiol. 2012;53(4):659-72.

35. Zouine M, Fu YY, Chateigner-Boutin A, Mila I, Frasse $\mathrm{P}$, Wang $\mathrm{H}$, et al, Characterization of the tomato ARF gene family uncovers a multi-levels post-transcriptional regulation including alternative splicing. Plos One. 2014;9:e84203.

36. Parapunova V, Busscher M, Busscher-Lange J, Lammers M, Karlova R, Bovy $A G$, et al. Identification, cloning and characterization of the tomato TCP transcription factor family. BMC Plant Biol. 2014;14:157.

37. Mayrose M, Ekengren SK, Melech-Bonfil S, Martin GB, Sessa G. A novel link between tomato GRAS genes, plant disease resistance and mechanical stress response. Mol Plant Pathol. 2006;7:593-604.

38. Marti C, Orzaez D, Ellul P, Moreno V, Carbonell J, Granell A. Silencing of DELLA induces parthenocarpy in tomato fruits. Plant J. 2007;52:865-76

39. Bassel GW, Mullen LT, Bewley JD. procera is a putative DELLA mutant in tomato (Solanum lycopersicum): effects on the seed and vegetative plant. J Exp Bot. 2008:59:585-93.

40. Jasinski S, Tattersall A, Piazza P, Hay A, Martinez-Garcia JF, Schmitz G, et al. PROCERA encodes a DELLA protein that mediates control of dissected leaf form in tomato. Plant J. 2008;56:603-12.

41. Tong HN, Jin Y, Liu WB, Li F, Fang J, Yin YH, et al. DWARF AND LOW-TILLERING, a new member of the GRAS family, plays positive roles in brassinosteroid signaling in rice. Plant J. 2009;58:803-16.

42. Llave C, Xie ZX, Kasschau KD, Carrington JC. Cleavage of scarecrow-like mRNA targets directed by a class of Arabidopsis miRNA. Science. 2002;297:2053-6.

43. Wang L, Mai YX, Zhang YC, Luo Q, Yang HQ. MicroRNA171C-targeted SCL6-II, SCL6-III, and SCL6-IV genes regulate shoot branching in Arabidopsis. Mol Plant. 2010;3:794-806.

44. de Jong M, Mariani C, Vriezen WH. The role of auxin and gibberellins in tomato fruit set. J Exp Bot. 2009;60:1523-32.
45. Kumar M, Khurana A, Sharma AK. Role of plant hormones and their interplay in development and ripening of fleshy fruits. J Exp Bot. 2014;65(16):4561-75

46. Jain M, Nijhawan A, Arora R, Agarwal P, Ray S, Sharma P, et al. F-box proteins in rice. Genome-wide analysis, classification, temporal and spatial gene expression during panicle and seed development, and regulation by light and abiotic stress. Plant Physiol. 2007;143:1467-83.

47. Aubourg S, Kreis M, Lecharny A. The DEAD box RNA helicase family in Arabidopsis thaliana. Nucleic Acids Res. 1999;27:628-36.

48. Jain M, Tyagi AK, Khurana JP. Genome-wide analysis, evolutionary expansion, and expression of early auxin-responsive SAUR gene family in rice Oryza sativa. Genomics. 2006;88:360-71.

49. Zou M, Guo B, He S. The roles and evolutionary patterns of intronless genes in deuterostomes. Comp Funct Genomics. 2011;2011:1-8.

50. Zhang D, lyer LM, Aravind L. Bacterial GRAS domain proteins throw new light on gibberellic acid response mechanisms. Bioinformatics 2012:28:2407-11.

51. Sun $X L$, Jones $W T$, Rikkerink E. GRAS proteins: the versatile roles of intrinsically disordered proteins in plant signaling. Biochem $J$ 2012;442:1-12

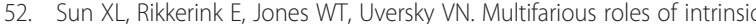
disorder in proteins illustrate its broad impact on plant biology. Plant Cell. 2013;25:38-55

53. Fode B, Siemsen T, Thurow C, Weigel R, Gatz C. The Arabidopsis GRAS protein SCL 14 interacts with class II TGA transcription factors and is essential for the activation of stress inducible promoters. Plant Cell. 2008;20:3122-35.

54. Hou XL, Lee YC, Xia KF, Yan Y, Yu H. DELLAs modulate jasmonate signaling via competitive binding to JAZs. Dev Cell. 2010;19:884-94.

55. Helariutta Y, Fukaki H, Wysocka-Diller J, Nakajima K, Jung J, Sena G, et al. The SHORT-ROOT gene controls radial patterning of the Arabidopsis root through radial signaling. Cell. 2000;101:555-67.

56. Fujisawa M, Shima Y, Higuchi N, Nakano T, Koyama Y, Kasumi T, et al. Direct targets of the tomato-ripening regulator RIN identified by transcriptome and chromatin immunoprecipitation analyses. Planta. 2012;235:1107-22.

57. Fujisawa M, Nakano $T$, Shima $Y$, Ito $Y$. A large-scale identification of direct targets of the tomato MADS box transcription factor RIPENING INHIBITOR reveals the regulation of fruit ripening. Plant Cell. 2013;25:371-86.

58. Santner A, Calderon-Villalobos LIA, Estelle M. Plant hormones are versatile chemical regulators of plant growth. Nature Chem Bio. 2009;5:301-7.

59. Gao MJ, Parkin IAP, Lydiate DJ, Hannoufa A. An auxin-responsive SCARECROW-like transcriptional activator interacts with histone deacetylase. Plant Mol Biol. 2004;55:417-31.

60. Rovere FD, Fattorini L, D'Angeli S, Veloccia A, Del Duca S, Cai G, et al. Arabidopsis SHR and SCR transcription factors and AUX1 auxin influx carrier control the switch between adventitious rooting and xylogenesis in planta and in in vitro cultured thin cell layers. Ann Bot. 2015;115:617-28.

61. Woodward AW, Bartel B. Auxin: regulation, action, and interaction. Ann Bot. 2005:95:707-35.

62. Peter H, Stephen GT. Gibberellin biosynthesis and its regulation. Biochem J. 2012;444:11-25.

63. Fujita M, Fujita Y, Noutoshi Y, Takahashi F, Narusaka Y, Yamaguchi-Shinozakl K, et al. Crosstalk between abiotic and biotic stress responses: a current view from the points of convergence in the stress signaling networks. Cur Opin Plant Biol. 2006;9:436-42.

64. Ma HS, Liang D, Shuai P, Xia XL, Yin WL. The salt- and drought-inducible poplar GRAS protein SCL7 confers salt and drought tolerance in Arabidopsis thaliana. J Exp Bot. 2010;61:4011-9.

65. Yang MG, Yang QY, Fu TD, Zhou YM. Overexpression of the Brassica napus BnLAS gene in Arabidopsis affects plant development and increases drought tolerance. Plant Cell Rep. 2011;30:373-88.

66. Achard P, Gong F, Cheminant S, Alioua M, Hedden P, Genschik P. The cold-inducible CBF1 factor-dependent signaling pathway modulates the accumulation of the growth-repressing DELLA proteins via its effect on gibberellin metabolism. Plant Cell. 2008;20:2117-29.

67. Jiang CF, Gao XH, Liao LL, Harberd NP, Fu XD. Phosphate starvation root architecture and anthocyanin accumulation responses are modulated by the gibberellin-DELLA signaling pathway in Arabidopsis. Plant Physiol. 2007;145:1460-70

68. Yao T, Bai SL, Li MM, Zhang YC, He YK. DELLA contribute to tolerance to nitric oxide stress in Arabidopsis seedlings. Chin Bull Bot. 2011;46:481-8. 
69. Bonshtien A, Lev A, Gibly A, Debbie P, Avni A, Sessa G. Molecular properties of the Xanthomonas AvrRxv effector and global transcriptional changes determined by its expression in resistant tomato plants. Mol Plant-Microbe Interact. 2005;18:300-10.

70. Mysore KS, Crasta OR, Tuori RP, Folkerts O, Swirsky PB, Martin GB. Comprehensive transcript profiling of Pto- and Prf-mediated host defense responses to infection by Pseudomonas syringae pv. tomato. Plant J. 2002;32:299-315.

71. Day RB, Tanabe S, Koshioka M, Mitsui T, Itoh H, Uequchi-Tanaka M, et al. Two rice GRAS family genes responsive to $\mathrm{N}$-acetylchitooligosaccharide elicitor are induced by phytoactive gibberellins: evidence for cross-talk between elicitor and gibberellin signaling in rice cells. Plant Mol Biol. 2004;54:261-72.

72. Xu K, Chen SJ, Li TF, Ma XS, Liang XH, Ding XF, et al. OsGRAS23, a rice GRAS transcription factor gene, is involved in drought stress response through regulating expression of stress-responsive genes. BMC Plant Biol. 2015;15:141.

73. Lehti-Shiu MD, Zou C, Hanada K, Shiu SH. Evolutionary history and stress regulation of plant receptor-like kinase/pelle genes. Plant Physiol. 2009;150:12-26

\section{Submit your next manuscript to BioMed Central and take full advantage of:}

- Convenient online submission

- Thorough peer review

- No space constraints or color figure charges

- Immediate publication on acceptance

- Inclusion in PubMed, CAS, Scopus and Google Scholar

- Research which is freely available for redistribution 